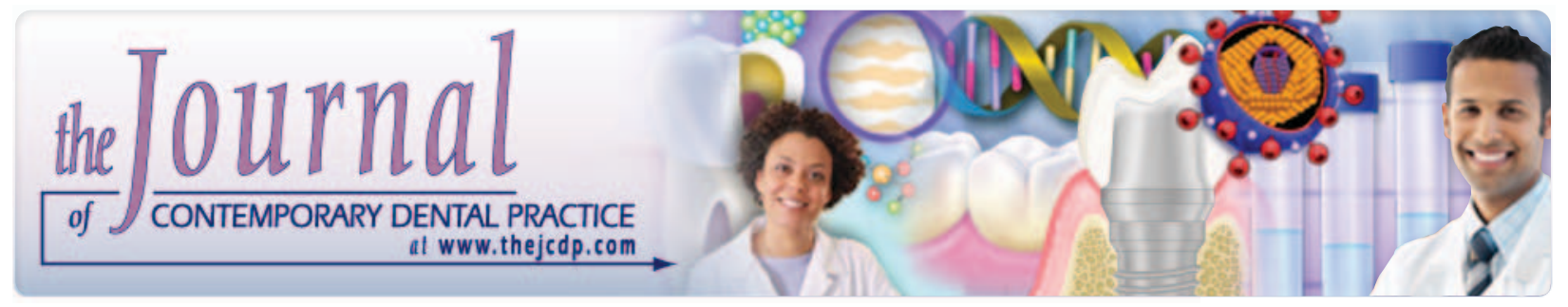

\title{
Endodontic Treatment of a Mandibular Second Premolar with Three Root Canals
}

Carlos Aguiar, BDS, MSc, PhD; Daniela Mendes, BDS; Andréa Câmara, BDS, MSc;

Jose Figueiredo, BDS, MSc, PhD

\section{Abstract}

Aim: The purpose of this case report is to describe a nonsurgical endodontic treatment of a mandibular left second premolar with two separate roots and three distinct root canals.

Background: In endodontics, the possible existence of extra canals must be considered before endodontic treatment is instituted. A wide morphological variation of the root canal system is known to exist.

Case Description: A 36-year-old male patient was referred for endodontic treatment on the left mandibular second premolar. Radiographic examination of the involved tooth revealed an unusual, complex root canal anato my. There was an irregular root morphology consisting of two distinct roots and three canals.

Summary: This case report describes the successful nonsurgical endodontic treatment of a mandibular left second premolar with two separate roots and three distinct root canals filled using size 35 Thermafil guttapercha carriers and $\mathrm{AH}$ Plus sealer. On the one-year follow-up radiograph, the tooth was asymptomatic, confirming adequate healing with no complications.

Clinical Significance: Even in a tooth with an extremely complex root canal morphology, conventional endodontic treatment without surgical intervention can result in adequate healing without any complications.

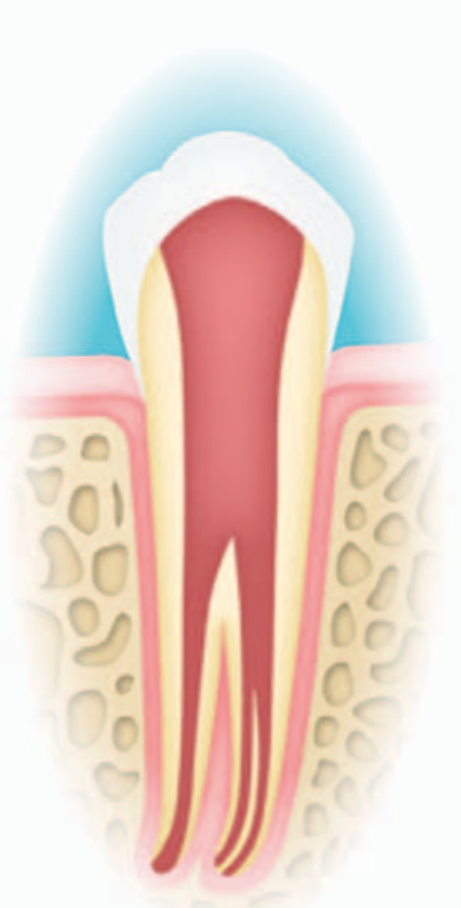

Keywords: Anatomic variations, endodontic treatment, mandibular second premolar, premolar, root canal morphology, Thermafil

Citation: Aguiar C, Mendes D, Câmara A, Figueiredo J. Endodontic Treatment of a Mandibular Second Premolar with Three Root Canals. J Contemp Dent Pract [Internet]. 2010 March; 11(2):078-084. Available from: http://www.thejcdp. com/journal/view/volume11-issue2-aguiar. 


\section{Introduction}

Complete cleaning and shaping is the key to successful endodontic treatment. A thorough understanding of the internal anatomy and morphology of the root canal system is an important consideration when performing cleaning and shaping procedures. ${ }^{1.2}$

In endodontics, the possible existence of extra canals must be considered before endodontic treatment is instituted. The clinician should always bear in mind that failure to identify an extranumerary canal might result in insufficient treatment and endodontic failure. Failure of endodontic treatment as a result of the inability to negotiate and instrumentate one of the additional canals is an important factor that may be encountered with mandibular premolars. ${ }^{3}$ Precise examination of root canal multiplicity on radiographs before treatment is essential to avoid missing canals. ${ }^{4}$

It has been reported that $2.5 \%$ of mandibular second premolars have two or more canals. ${ }^{5}$ Root canal morphology also can vary in different ethnic groups. ${ }^{2,6}$ This case describes the successful nonsurgical endodontic treatment of a mandibular left second premolar with two separate roots and three distinct root canals.

\section{Case Report}

A 36-year-old male patient with a noncontributory medical history was referred by his general dentist to the Endodontic Specialization Clinic at the Federal University of Pernambuco for endodontic treatment on the left mandibular second premolar.

The chief complaint of the patient was "pain in the left mandibular second premolar." Clinical examination revealed a temporary restoration in the left mandibular second premolar. The tooth was very sensitive to vertical percussion. Thermal and cavity pulp tests showed the pulp to be nonvital. Radiographic examination of the involved tooth revealed an unusual, complex root canal anatomy. There was an irregular root morphology consisting of two distinct roots and three canals (Figure 1). A diagnosis of pulp necrosis associated with acute apical periodontitis was made.
Following his consent, the tooth was anesthetized with $2 \%$ mepivacaine hydrochloride 1:100,000 epinephrine and isolated using a rubber dam; an access preparation was completed, after which the pulp chamber was irrigated with $1 \%$ sodium hypochlorite (Farmácia Escola Carlos Dumont de Andrade, Pernambuco, Brazil) and dried with a sterile cotton pellet. On entry into the pulp chamber, one main canal orifice was found that split into three different canal orifices at the midroot level. Exploration of the canal was carried out with the \#10 C+ file (Dentsply-Maillefer, Ballaigues, Switzerland) $5 \mathrm{~mm}$ short of the radiographic apex. The instrumentation technique used was the double-flared technique, using hand NiTi files (NitiFlex, Dentsply-Maillefer, Ballaigues, Switzerland) under constant irrigation. A freshly prepared $1 \%$ sodium hypochlorite solution (Roval, Recife, Brazil) was used in conjunction with ethylenediaminetetraacetic acid (Biodinâmica, Paraná, Brazil) as a chelating agent for irrigating the root canals. A working length, determined by radiography, was $18 \mathrm{~mm}$ for the mesial and 20 $\mathrm{mm}$ for the distobuccal and distolingual root canals (Figure 2). All root canals were enlarged to master file size of \#35 and dried with sterile absorbent paper points (Dentsply-Maillefer, Petrópolis, Brazil). A calcium hydroxide paste (Calen, SS White, Rio de Janeiro, Brazil) was applied, and access to the cavity was temporarily sealed with Cavit (3M ESPE, Seefeld, Germany). When the patient returned after one week, the calcium hydroxide paste was removed and the root canals were filled using size 35 Thermafil gutta-percha carriers (Dentsply Tulsa Dental Products, Tulsa,

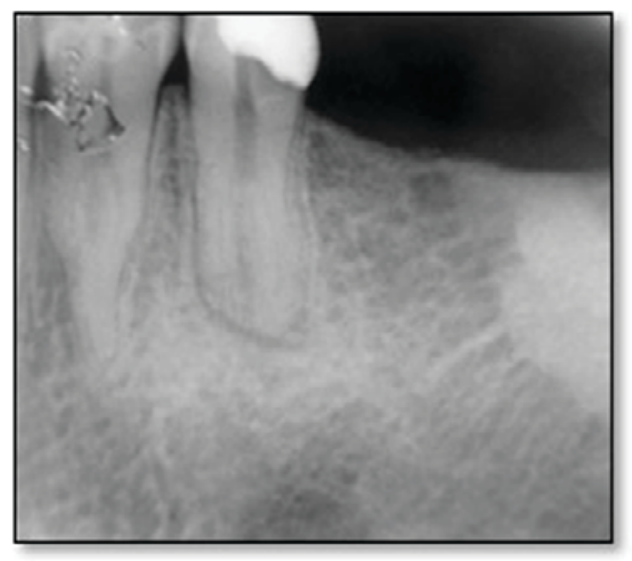

Figure 1. Preoperative periapical radiograph of the mandibular left second premolar. 
Oklahoma, USA) and AH Plus sealer (Dentsply/ DeTrey, Konstanz, Germany). A size 35 Thermafil verifier (Dentsply Tulsa Dental Products, Tulsa, Oklahoma, USA) was used to check the size of the canal; subsequently a thin layer of sealer was placed at the coronal orifice and the Thermafil gutta-percha was heated in a Thermaprep oven (Dentsply Tulsa Dental Products, Tulsa, Oklahoma, USA) according to the manufacturer's instructions. Firm apical pressure was used to insert the Thermafil Obturator to the working length. After the gutta-percha had cooled, a round diamond bur (KG Sorensen, Sao Paulo, Brazil) in a high-speed handpiece (Dabi-Atlante, Sao Paulo, Brazil) was used to cut the plastic shaft 1-2 mm inside the access cavity; the excess gutta-percha was then removed with a hot instrument and the pulp chamber cleaned with cotton balls soaked in orange oil (Phormula Ativa, Pernambuco, Brazil). A new radiograph showed that the root filling was complete (Figure $3)$. Finally, the crown was permanently restored with light-cured composite resin (TPH Spectrum, Dentsply, Rio de Janeiro, Brazil). On the one-year follow-up radiograph (Figure 4), the tooth was asymptomatic, confirming adequate healing with no complications.

\section{Discussion}

The extremely complex apical anatomy represents a considerable challenge when cleaning, shaping, and three-dimensionally obturating the root canal system are all required for endodontic treatment. ${ }^{-}$

Consistent, high levels of success in endodontic treatment require an understanding of root canal anatomy, morphology, and variations. To achieve endodontic success, the entire root canal system must be debrided, disinfected, and obturated. The clinician must have a thorough understanding of normal anatomy and of common variations from the norm. The clinician also must be prepared to identify those teeth that tend to vary greatly from the norm, e.g., mandibular premolars. ${ }^{4}$

Mandibular premolars have gained a reputation for having an aberrant anatomy. ${ }^{-}$Different studies have looked at the root canal morphology of mandibular premolars over the years and reported that a fairly high percentage of these teeth have more than one canal. $\frac{2,4-6,9,10}{\ln \text { a }}$

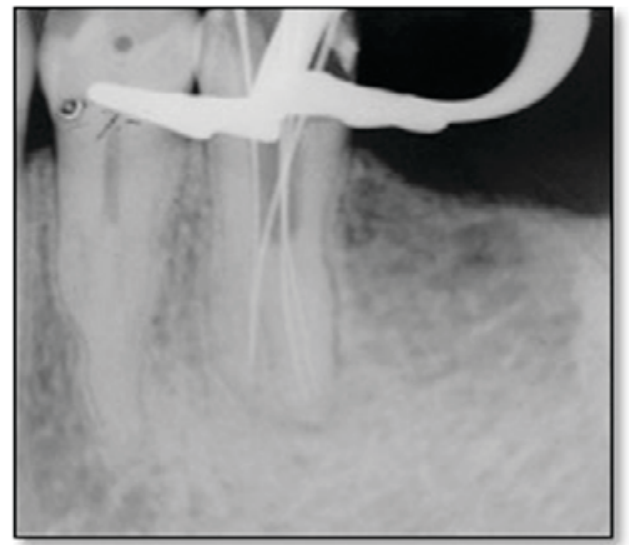

Figure 2. Working-length determination.

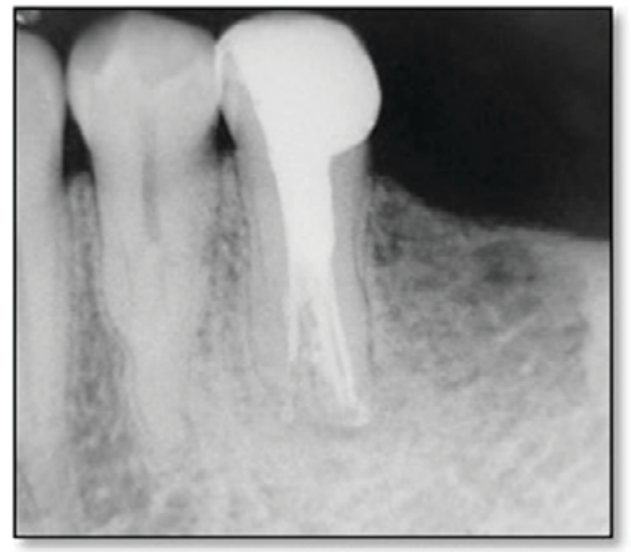

Figure 3. Immediate postoperative periapical radiograph taken after final canal obturation and placement of a coronal seal.

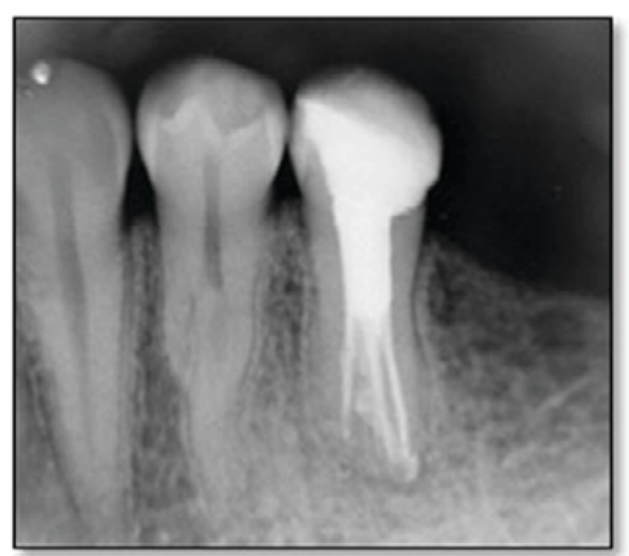

Figure 4. Twelve-month follow-up radiograph.

series of studies on extracted teeth, Vertucci $i^{\frac{5}{1}}$ in the 1970s and 1980s determined canal numbers and configurations by percentages for each of the teeth. He reported that the second premolars had only one root canal at the apex in $97.5 \%$ of the teeth studied and two canals in only $2.5 \%$; the incidence of three root canals was also low. 
Rödig and Hülsmann ${ }^{11}$ have reported a case of a mandibular second premolar with three separate roots and root canals diagnosed using intraoral periapical radiographs, as with this study, in which a mandibular left second premolar was observed with two separate roots and three distinct root canals diagnosed using intraoral periapical radiographs. Tzanetakis, Lagoudakos, and Kontakiotis; ${ }^{3}$ Sachdeva, Ballal, Gopikrishna, and Kandaswamy; ${ }^{9}$ Wong; ${ }^{12}$ Bram and Fleisher; ${ }^{13}$ and Al-Fouzan ${ }^{14}$ have reported the endodontic management of mandibular second premolars with four root canals.

In mandibular premolars with three canals, the cervical half of the root is generally wider than usual, with little or no taper. Root canals may not be evident radiographically or may look unusual. Root canal space may disappear halfway down the roots. Careful interpretation of the periodontal ligament space may suggest the presence of an extra root or canal. Mesial and distal angled views often will reveal the presence of a bi// trifurcation of the root canal. ${ }^{4}$

The problem with radiographs is that they produce only a two-dimensional image of a three-dimensional object, resulting in the superimposition of images. ${ }^{-}$Multiple canals originating from small pulp spaces are difficult to locate and even more difficult to treat. In this case, it was possible to locate and prepare all three canals, despite their originating at different levels, using intraoral periapical radiographs.

In the present case, after biomechanical preparation with hand instruments and copious irrigation with $1 \%$ sodium hypochlorite used in conjunction with ethylenediaminetetraacetic acid as a chelating agent, calcium hydroxide paste was chosen for the intracanal dressing; calcium hydroxide is helpful as an interappointment dressing because of its antimicrobial and tissuedissolving properties, thereby improving longterm success. ${ }^{15,16}$ Also, the use of intracanal medication may be needed to assist in the disinfection of root canal systems that are not fully accessible..$^{17}$

The key requirement for successful endodontic treatment is the complete removal of all irritants from the root canal system, followed by its obturation with a biocompatible material. For root canal filling, thermoplastic methods ${ }^{15}$ and a warm gutta-percha obturation technique $\frac{16,18,19}{\text { seem }}$ preferable to other techniques. The thermoplastic method was used in this case as an effective method of filling the irregular root canal space..$^{20,21}$ $\mathrm{AH}$ Plus is an epoxyamine-based resin and was chosen in this case as the root-end filling material for its favorable properties, such as an excellent sealing capacity, lower leakage, bond strength, good radiopacity, and bonding. $\frac{8,21,22}{}$

The diagnosis and management of extra roots or root canals in mandibular premolars is undoubtedly an endodontic challenge. In order to achieve success, the clinician must have a thorough understanding of the normal root canal anatomy and of its common variations. Inability to find and obturate a root canal has been shown to be a major cause of failure in endodontic therapy. $\stackrel{9}{-}$

\section{Summary}

A thorough examination of root canal multiplicity on radiographs before treatment is essential to prevent canals being overlooked. This case describes the nonsurgical endodontic treatment of a mandibular left second premolar with two separate roots and three distinct root canals. Root canal treatment was carried out successfully and the one-year follow-up radiograph confirmed adequate healing without any complications.

\section{Clinical Significance}

Even in a tooth with an extremely complex root canal morphology, conventional endodontic treatment without surgical intervention can result in adequate healing without any complications.

\section{References}

1. De Moor RJ, Calberson FL. Root canal treatment in a mandibular second premolar with three root canals. J Endod. 2005; 31(4):310-3.

2. Lu TY, Yang SF, Pai SF. Complicated root canal morphology of mandibular first premolar in a Chinese population using the cross section method. J Endod. 2006; 32(10):932-6.

3. Tzanetakis GN, Lagoudakos TA, Kontakiotis EG. Endodontic treatment of a mandibular second premolar with four canals using 
operating microscope. J Endod. 2007; 33(3):318-21.

4. Nallapati S. Three canal mandibular first and second premolars: a treatment approach. J Endod. 2005; 31(6):474-6.

5. Vertucci FJ. Root canal morphology of mandibular premolars. J Am Dent Assoc. 1978; 97(1):47-50.

6. Awawdeh LA, Al-Qudah AA. Root form and canal morphology of mandibular premolars in a Jordanian population. Int Endod J. 2008; 41(3):240-8.

7. Fava LR. The double-flared technique: an alternative for biomechanical preparation. J Endod. 1983; 9(2):76-80.

8. Plotino G, Grande NM, Pecci R, Bedini R, Pameijer $\mathrm{CH}$, Somma F. Three-dimensional imaging using microcomputed tomography for studying tooth macromorphology. J Am Dent Assoc. 2006; 137(11):1555-61.

9. Sachdeva GS, Ballal S, Gopikrishna V, Kandaswamy D. Endodontic management of a mandibular second premolar with four roots and four root canals with the aid of spiral computed tomography: a case report. J Endod. 2008; 34(1):104-7.

10. Cleghorn BM, Christie WH, Dong CC. The root and root canal morphology of the human mandibular second premolar: a literature review. J Endod. 2007; 33(9):1031-7.

11. Rödig $T$, Hülsmann M. Diagnosis and root canal treatment of a mandibular second premolar with three root canals. Int Endod J. 2003; 36(12):912-9.

12. Wong M. Four root canals in a mandibular second premolar. J Endod. 1991; 17(3):125-6.

13. Bram SM, Fleisher R. Endodontic therapy in a mandibular second bicuspid with four canals. J Endod. 1991; 17(10):513-5.

14. Al-Fouzan KS. The microscopic diagnosis and treatment of a mandibular second premolar with four canals. Int Endod J. 2001; 34(5): 406-10.

15. Steffen H, Splieth C. Conventional treatment of dens invaginatus in maxillary lateral incisor with sinus tract: one year follow-up. J Endod. 2005; 31(2):130-3.

16. Nallapati S. Clinical management of a maxillary lateral incisor with vital pulp and type 3 dens invaginatus: a case report. J Endod. 2004; 30(10):726-31.

17. da Silva Neto UX, de Moraes IG, Westphalen VP, Menezes R, Carneiro E, Fariniuk LF. Leakage of 4 resin-based root-canal sealers used with a single-cone technique. Oral Surg Oral Med Oral Pathol Oral Radiol Endod. 2007; 104(2):e53-7.

18. Rotstein I, Stabholz A, Heling I, Friedman S. Clinical considerations in the treatment of dens invaginatus. Endod Dent Traumatol. 1987; 3(5):249-54.

19. Xu Q, Ling J, Cheung GS, Hu Y. A quantitative evaluation of sealing ability of 4 obturation techniques by using a glucose leakage test. Oral Surg Oral Med Oral Pathol Oral Radiol Endod. 2007; 104(4):e109-13.

20. De-Deus G, Murad C, Paciornik S, Reis CM, Coutinho-Filho $T$. The effect of the canal-filled area on the bacterial leakage of oval-shaped canals. Int Endod J. 2008; 41(3):183-90.

21. Fisher MA, Berzins DW, Bahcall JK. An in vitro comparison of bond strength of various obturation materials to root canal dentin using a push-out test design. J Endod. 2007; 33(7):856-8.

22. Tanomaru-Filho M, Jorge EG, Guerreiro Tanomaru JM, Gonçalves M. Radiopacity evaluation of new root canal filling materials by digitalization of images. J Endod. 2007; 33(3):249-51.

\section{About the Authors}

\section{Carlos Aguiar, BDS, MSc, PhD} (Corresponding Author)

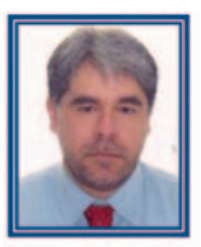

Dr. Aguiar is a Professor of Endodontics in the Department of Prosthetics and Oral and Facial Surgery, Faculty of Dentistry, at the Federal University of Pernambuco, in Recife, Brazil. His primary research interests include root canal preparation, rotary systems, biosafety, infection control, root canal anatomy, endodontics, endodontic sealers and root canal obturation.

e-mail: cmaguiar.ufpe@yahoo.com.br

\section{Daniela Mendes, BDS}

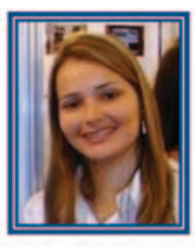

Dr. Mendes is a MSc student in Integrated Clinical Dentistry in the Department of Prosthetics and Oral and Facial Surgery, Faculty of Dentistry, at the Federal University of Pernambuco, in Recife, Brazil. 
Her primary research interests include root canal preparation, rotary systems, endodontics and root canal anatomy.

e-mail: mendes dam@ hotmail.com

Andréa Câmara, BDS, MSc

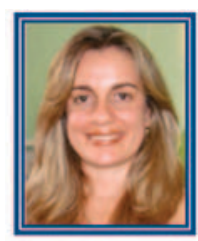

Dr. Câmara is a PhD student in Pharmaceutical Sciences in the Department of Pharmaceutical Sciences at the Federal University of Pernambuco, in Recife, Brazil. Her primary research interests include root canal irrigants,

microbiology, rotary systems, root canal preparation and endodontics.

e-mail: andreaccam@yahoo.com.brl

Jose Figueiredo, BDS, MSc, PhD

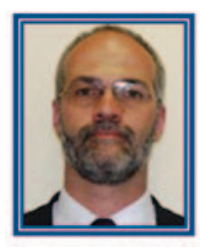

Dr. Figueiredo is a Reader in Endodontology, Dean of the PostGraduate Program in Dentistry at the Pontifical Catholic University of Rio Grande do Sul - PUCRS, in Porto Alegre, Brazil. His primary research interests include microbiology, root canal preparation, rotary systems, root canal anatomy, endodontics, endodontic sealers and root canal obturation.

e-mail: jose.figueiredo@pucrs.br 